\title{
Growth of high-density horizontally aligned SWNT arrays using Trojan catalysts
}

Yue $\mathrm{Hu}^{1, \star}$, Lixing Kang ${ }^{1,2, \star}$, Qiuchen Zhao ${ }^{1, \star}$, Hua Zhong ${ }^{3}$, Shuchen Zhang ${ }^{1}$, Liangwei Yang ${ }^{1}$, Zequn Wang ${ }^{1}$, Jingjing Lin ${ }^{1}$, Qingwen Li ${ }^{2}$, Zhiyong Zhang ${ }^{3}$, Lianmao Peng ${ }^{3}$, Zhongfan Liu' \& Jin Zhang ${ }^{1}$

Single-walled carbon nanotube (SWNT)-based electronics have been regarded as one of the most promising candidate technologies to replace or supplement silicon-based electronics in the future. These applications require high-density horizontally aligned SWNT arrays. During the past decade, significant efforts have been directed towards growth of high-density SWNT arrays. However, obtaining SWNT arrays with suitable density and quality still remains a big challenge. Herein, we develop a rational approach to grow SWNT arrays with ultra-high density using Trojan catalysts. The density can be as high as 130 SWNTs $\mu \mathrm{m}^{-1}$. Field-effect transistors fabricated with our SWNT arrays exhibit a record drive current density of $-467.09 \mu \mathrm{A} \mathrm{m}^{-1}$ and an on-conductance of $233.55 \mu \mathrm{S} \mu \mathrm{m}^{-1}$. Radio frequency transistors fabricated on these samples exhibit high intrinsic $f_{\mathrm{T}}$ and $f_{\mathrm{MAX}}$ of 6.94 and $14.01 \mathrm{GHz}$, respectively. These results confirm our high-density SWNT arrays are strong candidates for applications in electronics.

\footnotetext{
${ }^{1}$ Center for Nanochemistry, Beijing National Laboratory for Molecular Sciences, State Key Laboratory for Structural Chemistry of Unstable and Stable Species, College of Chemistry and Molecular Engineering, Peking University, Beijing 100871, China. ${ }^{2}$ Advanced Materials Division, Suzhou Institute of Nanotech and Nanobionics, Chinese Academy of Science, Suzhou 215123, China. ${ }^{3}$ Department of Electronics, Key Laboratory for the Physics and Chemistry of Nanodevices, Peking University, Beijing 100871, China. ${ }^{\star}$ These authors contributed equally to this work. Correspondence and requests for materials should be addressed to J.Z. (email: jinzhang@pku.edu.cn).
} 
$\mathrm{n}$ the past 20 years, marked advances in single-walled carbon nanotube (SWNT)-based integrated circuits (ICs) have been witnessed $^{1,2}$. However, the density and quality of SWNT arrays still limit the performance of devices. In 2013, the scientists of IBM projected that, the desired density of SWNT arrays should be higher than 125 SWNTs $\mu \mathrm{m}^{-1}$ to realize the SWNT-based IC applications ${ }^{3}$. Several methods have been developed to produce high-density SWNT arrays, including post-treatment approaches and in situ growth methods ${ }^{4-12}$. In the case of post-treatment approaches, the multiple transfer method could obtain SWNT arrays with the density as high as 55 SWNTs $\mu \mathrm{m}^{-1}$ (ref. 5). However, these transferred SWNTs are not as aligned as asgrown SWNTs, which could lead to increased tube-to-tube interactions and degenerate the SWNTs' quality. Meanwhile, Langmuir-Blodgett ${ }^{6}$ and Langmuir-Schaefer ${ }^{7}$ methods can also be utilized to increase the density of SWNT arrays, which can reach 500 SWNTs $\mu \mathrm{m}^{-1}$. Nevertheless, post-treatment inevitably brought contamination, and the tubes were very short and in poor orientation, which cannot meet the requirement of high-performance devices. For the in situ growth methods, especially in chemical vapour deposition (CVD) systems, multiple loading catalyst ${ }^{8}$, multiple-cycle growth ${ }^{9}, \mathrm{CuCl}_{2}$ /polyvinylpyrrolidone alcohol solution growth ${ }^{4}$ and periodic growth ${ }^{10}$ have been developed and the density can reach 10-50 SWNTs $\mu \mathrm{m}^{-1}$. These SWNT arrays show good qualities and perfect alignment, but densities are still not high enough for SWNT-based IC applications ${ }^{3,11}$.

To ensure SWNT quality and alignment, in situ growth of highdensity SWNT arrays has many advantages. In the CVD process, the catalyst is a key factor for growing SWNTs on substrates ${ }^{12-14}$. How to maintain the activity of catalysts ${ }^{15,16}$, give catalyst nanoparticles more opportunities to nucleate SWNTs, ${ }^{9,17}$ and provide new catalysts during SWNT growth ${ }^{8}$ are key issues for obtaining SWNT arrays with ultra-high density on substrates. In this study, ultra-high density of SWNT arrays were realized through use of Trojan catalysts. Figure 1a schematically illustrates the procedure of the approach. First, the catalyst's precursor was loaded onto the substrate by spin coating. After a high-temperature annealing process, the catalysts dissolve into the substrate and then become stored. Catalysts can then be released gradually under hydrogen atmosphere during growing process. As the gradual release mode reduces the interaction between active catalysts, which could lead to the deactivation of catalysts ${ }^{18-21}$, our growth mode obviously increases the efficiency of catalysts, and realizes the ultra-high density of SWNT arrays. The substrate acts as a catalyst container and the catalysts hide in the container. We named these catalysts as Trojan catalysts by analogy with soldiers emerging from the Trojan horse in the Greek story.

\section{Results}

Characterization of high-density SWNT arrays. From scanning electron microscope (SEM) observations (Fig. 1b,c), uniform ultra-high-density SWNT arrays with large area were obtained using Trojan catalysts. Figure $1 \mathrm{c}$ shows an area with $1 \mu$ width. The average nanotube density is measured to be at least 130 tubes $\mu \mathrm{m}^{-1}$ based on the number of the black lines, which were taken as SWNTs in the SEM image ${ }^{22}$. Higher-magnification SEM and atomic force microscope (AFM) images (insets in Fig. 1c) further clearly indicate the density of the as-grown horizontally aligned SWNT arrays is as high as 130 SWNTs $\mu \mathrm{m}^{-1}$. This is the highest reported density of grown SWNT arrays to date. Raman spectra of the high-density SWNT arrays with $514.5 \mathrm{~nm}$ excitation (Fig. 1d) show a distribution of radial breathing mode peaks between 110 and $190 \mathrm{~cm}^{-1}$ and the barely noticeable D band at a high-frequency region, which a
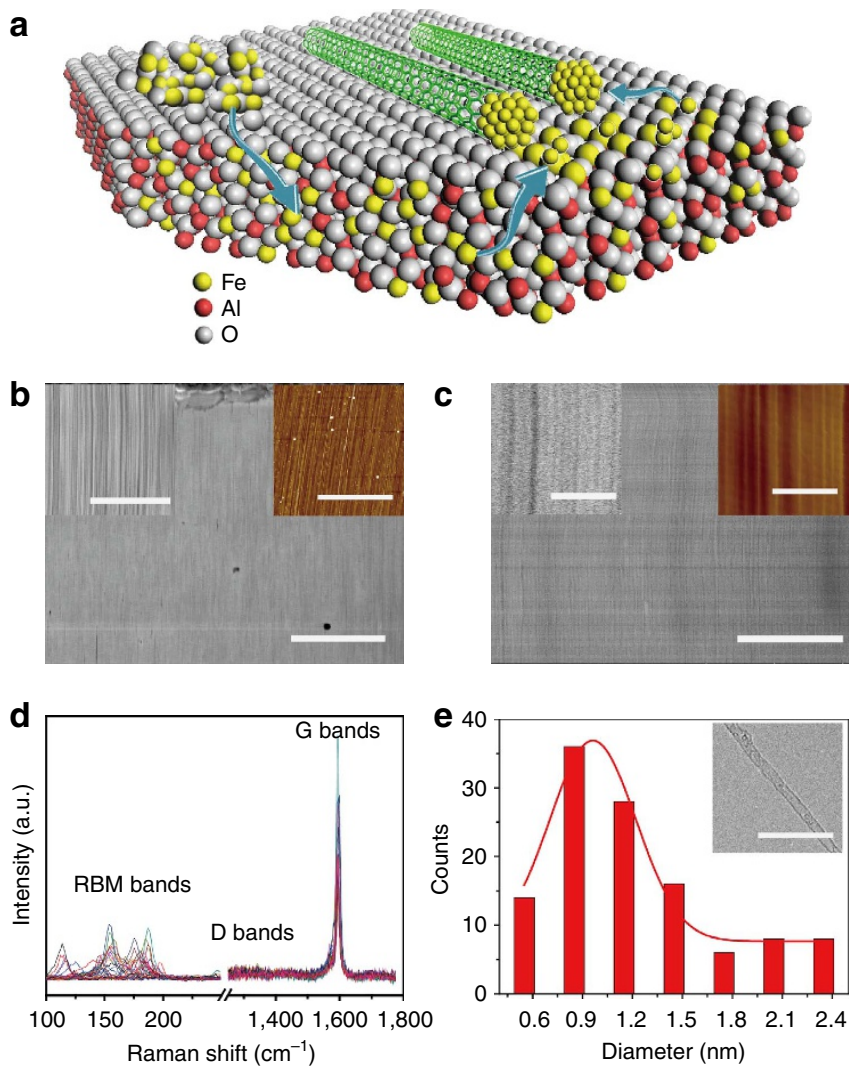

Figure 1 | Schematic illustration of growth mechanism and characterization of high-density SWNT arrays. (a) Schematic illustration of growing high-density horizontally aligned single-walled carbon nanotube arrays using Trojan catalysts. (b,c) SEM and AFM images of the as-grown SWNT arrays on sapphire surface at low and high magnifications, respectively, (insets: left SEM images, right AFM images). AFM and SEM images (insets in c) at higher magnification confirm that the density of SWNT arrays is as high as 130 SWNTs $\mu m^{-1}$. (d) Raman spectra of the as-grown SWNT with $514.5 \mathrm{~nm}$ excitation. (e) Diameter distribution of the as-grown SWNT arrays (insets: HRTEM image of an individual SWNT). The red solid lines are Gaussian fitting peaks. Scale bar, $50 \mu \mathrm{m}$ (b) (scale bar, $3 \mu \mathrm{m}$ for the insets in b). Scale bar, $300 \mathrm{~nm}$ (c) (scale bar, $50 \mathrm{~nm}$ for the insets in c). Scale bar, $10 \mathrm{~nm}(\mathbf{e})$.

indicate that the aligned SWNT arrays are of high quality. Highresolution transmission electron microscopy observations on the as-grown sample revealed that only SWNTs were found (inset in Fig. 1e). We observe that arrays grown in this fashion always consist of individual SWNTs, which is consistent with those reported in literature ${ }^{23,24}$. Furthermore, AFM measurements (Fig. 1e) show the main diameter distribution of the SWNT arrays from 0.8 to $1.6 \mathrm{~nm}$ with an average diameter of $1.26 \mathrm{~nm}$.

The dissolve-and-release mechanism for Trojan catalysts. To realize the growth of SWNT arrays with high density, one possibility is to change the loading method of catalyst precursors onto the substrate. Loading catalyst precursors over time has been proved to be useful in high-density growth ${ }^{8}$. The key point of these methods is that newly loaded catalysts can be active during the SWNT growth process, which increases the density of asgrown SWNT arrays. To further improve the density of catalysts, we first proposed the continuous formation of catalyst nanoparticles during SWNT growth through a novel catalyst design. We attempted to hide the catalyst precursor inside the substrate before the CVD growth process. Under proper 
a

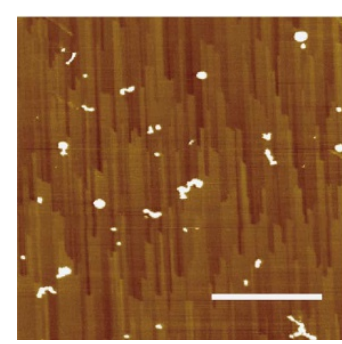

d

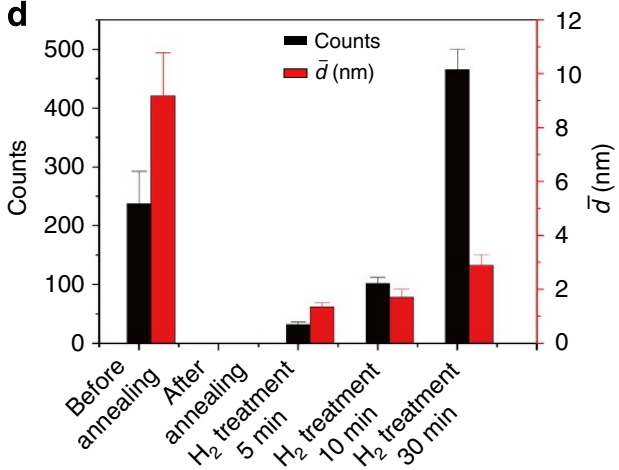

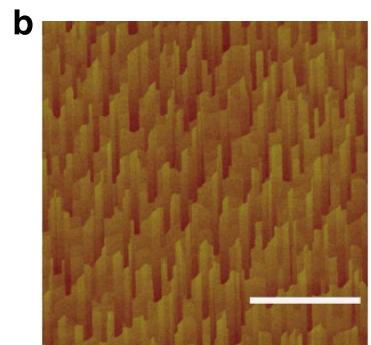

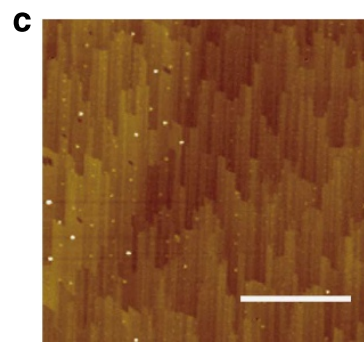

e

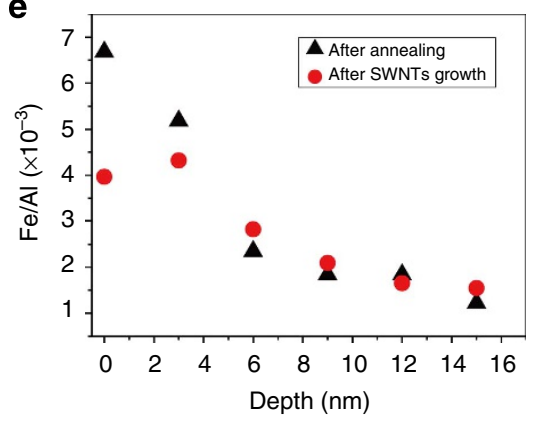

Figure 2 | Verification of the catalyst-dissolving and -releasing processes. (a) AFM images of substrate after spin-coating Fe(OH) 3 alcohol solution. (b) AFM images of sapphire substrate with loaded catalysts after annealing in a meffle furnace. (c) AFM images of substrate after $\mathrm{H}_{2}$ treatment for 30 min. (d) The counts and average diameters of catalyst particles statistics in the areas ( $3 \mu \mathrm{m} \times 3 \mu \mathrm{m})$ after different treatment conditions: before and after annealing, $\mathrm{H}_{2}$ treatment for 5, 10 and $30 \mathrm{~min}$, respectively. The error bars of counts were s.d. that were calculated from five time statistic results.

(e) XPS depth analysis of the substrates after annealing and after SWNTs growth. Scale bar, $1 \mu \mathrm{m}$.

conditions, the catalyst precursor should be able to release from the substrate to form catalyst nanoparticles over time. We noted that the structure of sapphire $\left(\alpha-\mathrm{Al}_{2} \mathrm{O}_{3}\right)$ and haematite $\left(\alpha-\mathrm{Fe}_{2} \mathrm{O}_{3}\right)$ are very similar, as well as the diameter of low-spin $\mathrm{Fe}^{3+}$ and $\mathrm{Al}^{3+}$ (more details are given in Supplementary Fig. 1 and Supplementary Table 1). This indicates that $\mathrm{Al}^{3+}$ can be replaced partly by $\mathrm{Fe}^{3+}$ in the sapphire substrate. By a simple thermodynamic analysis in Supplementary Fig. 2, this process is driven by entropy, for the chaos of the system will increase during the inter-infiltration between the adjacent crystal surfaces, until the components of these two surfaces verge to the same. To verify our assumption, $\mathrm{Fe}(\mathrm{OH})_{3}$ /ethanol solution was deposited on the surface of the sapphire substrate by spin coating. Then the substrate was treated by $1,100{ }^{\circ} \mathrm{C}$ annealing for $8 \mathrm{~h}$. Figure $2 \mathrm{a}$ and Fig. $2 \mathrm{~b}$ show the AFM analysis of the surface morphology of sapphire before and after annealing process, respectively. The particles on the surface in Fig. 2a, which were known to be $\mathrm{Fe}(\mathrm{OH})_{3}$ nanoparticles, were not found in Fig. 2b. To ensure that $\mathrm{Fe}$ (III) had dissolved into the bulk phase of sapphire instead of being volatilized during the annealing process, we performed depth profiling after removing the surface layer by argon bombardment, which is given in Fig. 2e. The content of Fe(III) had an exponential decrease with increasing depth in the substrate. To exclude the possibility that the sputtering itself causes the diffusion of the iron precursor, we loaded the catalyst precursor on sapphire by spin coating, as shown in Fig. 2a, and then performed X-ray photoelectron spectroscopy (XPS) depth profiling without any annealing process. The result is shown in Supplementary Fig. 3. From what we can see, only the first plot shows a relatively high percentage of Fe. Fe signals are too weak to be observed in deeper sites. These results can be a strong evidence to provide strong evidence that Fe(III) has dissolved into the substrate.

As we all know, $\mathrm{Fe}_{2} \mathrm{O}_{3}$ can be reduced to $\mathrm{Fe}$ by $\mathrm{H}_{2}$ treatment, but $\mathrm{Al}_{2} \mathrm{O}_{3}$ cannot. Thus, dissolved $\mathrm{Fe}(\mathrm{III})$ could be reduced to
Fe and form nanoparticles on the surface, which could act as catalysts to grow high-density SWNT arrays. To ensure that dissolved Fe(III) could afford the requirements of catalyst nanoparticles in high-density SWNT arrays, we deeply analysed the XPS data, as shown in Supplementary Fig. 4. We found that only $1.54 \%$ of the $\mathrm{Al}^{3+}$ was replaced by $\mathrm{Fe}^{3+}$ in the first layer of sapphire. Meanwhile, Supplementary Fig. 5 showed that if $0.1 \%$ of the $\mathrm{Fe}$ (III) in the first layer was reduced to $\mathrm{Fe}$ and formed the nanoparticles, we can get a high-density horizontal SWNT array with 150 SWNTs $\mu \mathrm{m}^{-1}$ in density and $200 \mu$ in length. Furthermore, Fe was not a unique case of the Trojan catalysts. Similar phenomena were observed in Fe, Mn and Co, but not found in Mo and $\mathrm{Cu}$ (more details are shown in Supplementary Fig. 6 and Supplementary Table 2). Most ions with similar diameters and same valence as $\mathrm{Al}^{3+}$ can dissolve into sapphire, which could be regarded as an extension of the Trojan catalysts.

Under our CVD growth condition at $830^{\circ} \mathrm{C}, \mathrm{Fe}_{2} \mathrm{O}_{3}$ can be reduced to $\mathrm{Fe}$ in hydrogen ambience ${ }^{25}$. $\mathrm{Fe}_{2} \mathrm{O}_{3}$ nanoparticles without any treatment can be reduced rapidly. In our Trojan catalysts, Fe(III) was dissolved into the substrate, which decreased the contact area between $\mathrm{Fe}$ (III) and $\mathrm{H}_{2}$. Thus, the reduction rate slows down intuitively. This ensures a continuous supply of Fe and realizes the gradual formation of the catalysts. Figure $2 \mathrm{c}$ shows the AFM analysis of the sapphire surface after a 30-min treatment with $\mathrm{H}_{2}$. Nanoparticles can be observed to be denser than those in Fig. 2b. Before the annealing process, the diameters of the particles were larger and the shapes of the particles were anomalous. After an annealing and reduction process, the nanoparticles became smaller and denser with a more regular geometrical shape, which was more suitable to act as catalysts. We also studied the relationship between reduction time and the topography of catalyst nanoparticles. Figure $2 \mathrm{~d}$ shows the areal density (bars in black) and the diameter (bars in red) variation for the different treatment times with $\mathrm{H}_{2}$. We can see that with the additional reduction time, the diameter of the nanoparticle 

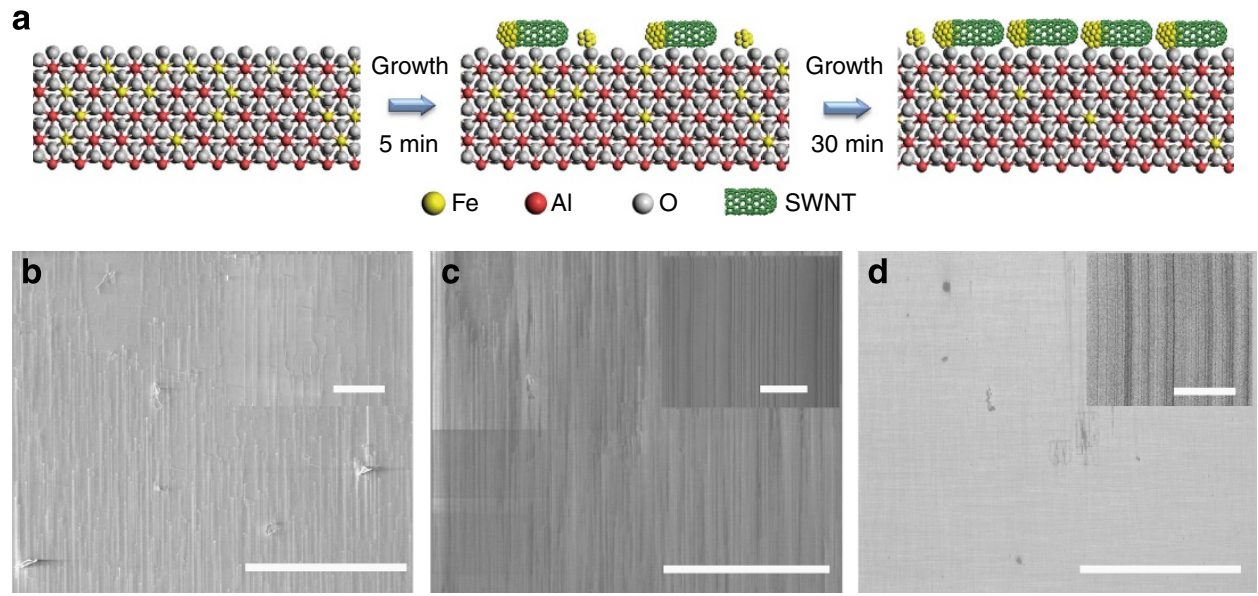

Figure 3 | The density of SWNTs increases with the growth time extension. (a) Schematic illustration of catalysts gradually released and catalysed the constant growth of SWNTs in the CVD process. (b-d) SEM images of SWNTs grown from the same catalyst area (marked with FFB) after the first CVD growth at $5 \mathrm{~min}(\mathbf{b})$, after the second CVD growth at $10 \mathrm{~min}$ (c) and after the third CVD growth at 30 min (insets: high-magnification SEM images). Scale bar, $100 \mu \mathrm{m}$ (b-d) (inset in $\mathbf{b}$ is $20 \mu \mathrm{m}$, inset in $\mathbf{c}$ is $1 \mu \mathrm{m}$ and inset in $\mathbf{d}$ is $200 \mathrm{~nm}$ ).

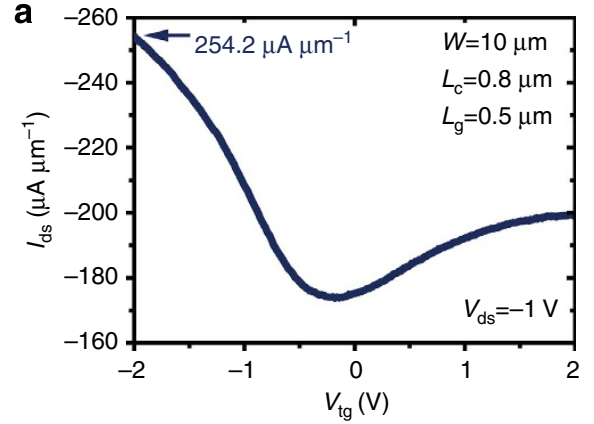

C

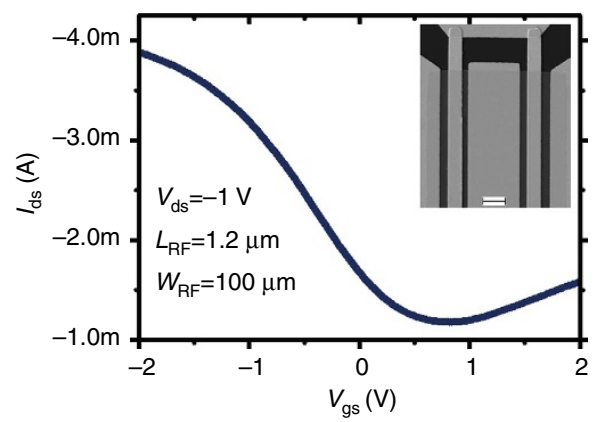

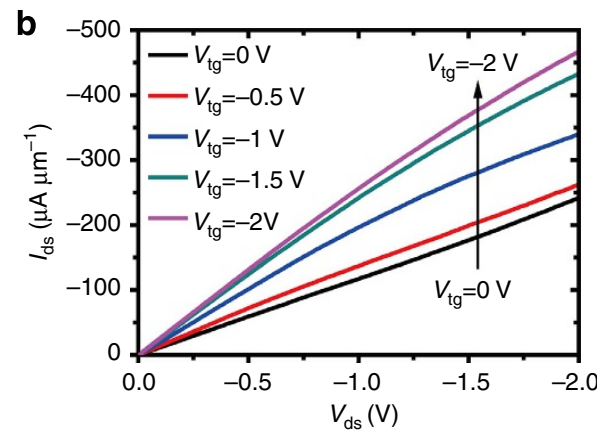

d
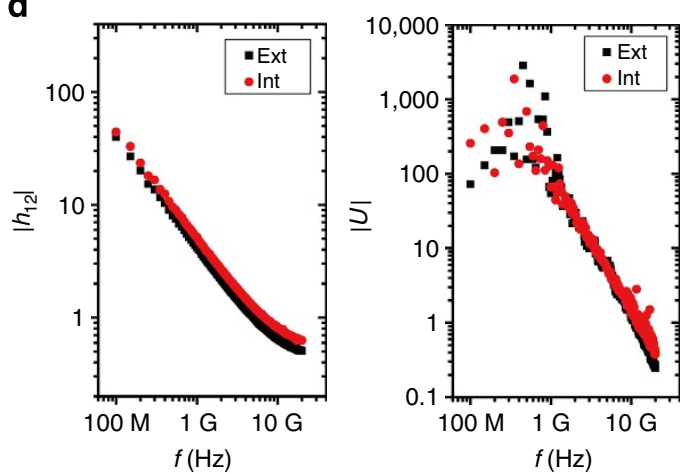

Figure 4 | High performance of high -density SWNT arrays devices. (a) Transfer characteristics of a high-current density device. $V_{\mathrm{ds}}=-1 \mathrm{~V}$. (b) Output characteristics of the high-current density devices. $V_{\text {tg }}$ varies from 0 to $-2.0 \mathrm{~V}$ in steps of $0.5 \mathrm{~V} . \mathrm{W}=10 \mu \mathrm{m}, L_{\mathrm{ch}}=0.8 \mu \mathrm{m}, L_{\mathrm{g}}=0.5 \mu \mathrm{m}$. (c) Transfer characteristics of the RF transistor. Inset: SEM of the RF transistor. Scale bar, $2 \mu \mathrm{m}$. $W_{\mathrm{RF}}=2 \times 50 \mu \mathrm{m}, L_{\mathrm{RF}}=1.2 \mu \mathrm{m}$ and $V_{\mathrm{ds}}=-1 \mathrm{~V}$. (d) The extrinsic (black dots) and intrinsic (red dots) current gain $\left|h_{21}\right|$ and power gain $|U|$ as a function of frequency.

changed not obviously, while the areal density of the nanoparticles increases distinctly (more details are shown in Supplementary Fig. 7). The phenomenon could be a good proof that when Fe (III) are dissolved into the sapphire substrate, the reduction rate slows down obviously. Furthermore, the dissolveand-release process also increases the density of catalysts and decreases the diameter of catalyst nanoparticles. To analyse the reduction process, especially the reaction site in the sapphire substrate, we performed another XPS depth analysis after the growth process, which is shown in Fig. 2e. We could see that the surface content of Fe (III) decreases significantly. This could be considered as the volatilization during $\mathrm{H}_{2}$ treatment under high temperature during nanotube growth. The component of the substrate in a deeper area $(>3 \mathrm{~nm})$ remains, which means catalyst release, or $\mathrm{Fe}(\mathrm{III})$ reduction, mostly happened on the surface of the substrate (more details about XPS analysis are shown in Supplementary Fig. 8). These phenomena confirmed that $\mathrm{H}_{2}$ treatment can release Fe from substrate gradually, which agreed with our catalyst design.

To verify our hypothesis that the gradually released catalysts in the CVD process resulted in the increasing density of SWNTs, which is schematic illustrated in Fig. 3a, we performed three 
growth processes respectively on the same substrate under different CVD growth time. The sample was taken out from the furnace after the first growth of $5 \mathrm{~min}$. After SEM characterization and annealing at $1,100^{\circ} \mathrm{C}$ in air for $8 \mathrm{~h}$, the same sample was put back into the furnace for the second growth under the same CVD conditions for $10 \mathrm{~min}$. A third growth was performed the same as the second one, except the growth time was $30 \mathrm{~min}$ instead of $10 \mathrm{~min}$. We compared the growth results in the same region with the help of marks on the sapphire substrate after every growth process. SEM images (Fig. 3b-d) showed the density of SWNT arrays gradually increased from $<1$ SWNTs $\mu \mathrm{m}^{-1}$ after the first growth to about 10 SWNTs $\mu \mathrm{m}^{-1}$ after the second growth and then reached the highest value (130 SWNTs $\mu \mathrm{m}^{-1}$ ) after the third growth. The density of SWNTs increased with the growth time extension, which means that new active catalysts formed continuously and catalysed the growth of new SWNTs. Furthermore, we attempted to grow SWNTs with other kinds of metals, such as Co and Mn, which were members of Trojan catalysts. As shown in Supplementary Fig. 9, the density of the SWNT arrays can be increased with an annealing process after loading the catalyst than that without annealing. Differences among each metal in the density of arrays came from differences in the catalytic efficiency. This proved the universality of our Trojan catalysts. In addition, we utilized ion implantation technology to let the catalyst enter into the substrate ${ }^{26}$ and successfully obtained high-density-aligned SWNT arrays as well (Supplementary Fig. 10). This also confirmed the dissolving and releasing processes of Trojan catalysts. Thus, we believe that the gradually released catalysts are significant to obtaining ultra-highdensity SWNT arrays.

Electrical properties of high-density SWNT arrays. Experimental and theoretical works have demonstrated that the densealigned SWNT arrays are important and desirable for the applications of nanotube-based electronics, such as ICs and radio frequency (RF) electronics 27,28 . To evaluate the electrical performance of such ultra-high-density-aligned SWNT arrays, top-gated field effect transistors (FET) based on our as-grown SWNT arrays were fabricated directly on the growth substrate, and the electrical transport measurements were carried out in both d.c. and a.c. domains. Figure 4a,b present the d.c. electrical characterization of the fabricated FETs, where the channel length $L_{\mathrm{ch}}$ is $0.8 \mu \mathrm{m}$, gate length $L_{\mathrm{g}}$ is $0.5 \mu \mathrm{m}$, and channel width $W$ is $10 \mu \mathrm{m}$. It is seen that the largest on-state current could even reach $-467.09 \mu \mathrm{A \mu m}{ }^{-1}$ at $V_{\mathrm{ds}}=V_{\mathrm{gs}}=-2 \mathrm{~V}$, corresponding to an on-conductance of $233.55 \mu \mathrm{S} \mu \mathrm{m}^{-1}$. As we know, the current density, or on-conductance, is the best results ever reported based SWNT arrays at the same scale of our channel length, and even match the results of solution-based SWNT arrays with a nanotube density of more than 500 tubes per $\mu \mathrm{m}$, although the channel length of our device is almost 10 times larger ${ }^{7,29}$. This remarkably high current density and transconductance derives from the high density and perfect quality of our as-grown SWNT arrays. To further demonstrate the superiority of our high-density and quality SWNT arrays, we also measured the high-frequency performance of the fabricated RF transistors with $L_{\mathrm{g} 2}=1.2 \mu \mathrm{m}$ and $W_{2}=100 \mu \mathrm{m}$. Transfer characteristics of RF FETs are presented in Fig. 4c, where the SEM of the device structure was displayed as an inset. As shown in Fig. $4 \mathrm{~d}$, the extrinsic cutoff frequency $f_{\mathrm{T}}$ and maximum oscillation frequency $f_{\mathrm{MAX}}$ achieved are 5.13 and $11.33 \mathrm{GHz}$, respectively, which are comparable to the best performance of SWNT array-based RF transistors ${ }^{28}$. The intrinsic $f_{\mathrm{T}}$ and $f_{\mathrm{MAX}}$ of our RF transistor are 6.94 and $14.01 \mathrm{GHz}$, respectively, after the de-embedding process. As $f_{\mathrm{T}}$ is associated with short-circuit current gain and $f_{\text {MAX }}$ reflects the unilateral power gain, the latter is a much more important and practical figure of merit in analogue circuit application. The highest intrinsic $f_{\text {MAX }}$ of an SWNT array-based transistor is about $30 \mathrm{GHz}$, where the channel length is about $100 \mathrm{~nm}$. Therefore, our $1.2-\mu \mathrm{m}$-length transistors with an intrinsic $f_{\mathrm{MAX}}$ of $14.01 \mathrm{GHz}$ reveals a great potential for application of in situ grown SWNT arrays in the future RF electronics.

\section{Discussion}

In summary, a facile and effective strategy for growing highdensity SWNT arrays was developed. We could obtain ultra-highdensity $\left(>130\right.$ SWNTs $\left.\mu \mathrm{m}^{-1}\right)$ horizontally aligned SWNT arrays through the use of Trojan catalysts, which is the highest density of grown SWNTs ever reported. Systematic experiments were performed to verify the catalyst-dissolving and -releasing processes by AFM, XPS, SEM and ion implantation technology. In addition, RF circuits based on the Trojan catalyst-synthesized nanotubes could have great potential for SWNT applications in future nanoelectronics. Trojan catalysts are useful for understanding the mechanism of SWNTs growth as well. Furthermore, combining s-SWNT arrays growth with Trojan catalysts, desired s-SWNT arrays with high density will be achieved. Furthermore, Trojan catalysts provide a new strategy for SWNT growth, but are not limited to SWNT growth. The essence of our Trojan catalysts is to release catalysts gradually and further to increase the efficiency, which is not limited to any kind of particular category of catalysts nor the growth of SWNT arrays with high density. It should be taken as a universal method and should be applicable for the catalyst-assisted fabrication of other one-dimensional nanomaterials, such as $\mathrm{Si}, \mathrm{Ge}, \mathrm{GaN}$ and $\mathrm{ZnO}$ nanowires or nanotubes and so on.

\section{Methods}

Synthesis of high-density-aligned SWNT arrays. In particular, a-plane sapphire substrates (single-side polished, miscut angle $<0.5^{\circ}$, surface roughness $<5 \AA$ ) were purchased from Hefei Kejing Materials Technology Co., China. After cleaning, the sapphire substrates must undergo a necessary annealing process (at $1,100{ }^{\circ} \mathrm{C}$ in air for $8 \mathrm{~h}$ ) for better crystallization (see Supplementary Fig. 11). A total of

$0.05 \mathrm{mmoll}^{-1} \mathrm{Fe}(\mathrm{OH})_{3}$ /ethanol solution, which was used as a catalyst precursor, was dispersed onto the sapphire substrates by the spin-coating method. Then, the sapphire substrates with iron species on the surface were followed by a second annealing process (at $1,100^{\circ} \mathrm{C}$ in air for $8 \mathrm{~h}$ as the same as stated above) for the iron species to dissolve into the sapphire substrates. The SWNT growth was performed in a furnace with a 1-inch tube. The sapphire substrates with well-dispersed iron species were put into the tube and heated in air to the desired temperature. After the system was purged with 300 standard cubic centimeters per minute (s.c.c.m.) argon, a flow of hydrogen (100 s.c.c.m.) and argon (50 s.c.c.m., through an ethanol bubbler) were introduced for ultra-high-density growth SWNT arrays at $830^{\circ} \mathrm{C}$ for $30 \mathrm{~min}$.

Combining with ion implantation technology. The clean a-plane sapphire substrates were annealed at $1,100^{\circ} \mathrm{C}$ in air for $8 \mathrm{~h}$ in advance, then were implanted at room temperature with a mass-analysed beam of $\mathrm{Fe}$ ions $(15 \mathrm{keV})$ to fluences of $1 \times 10^{15}$ to $1 \times 10^{16} \mathrm{Fe} \mathrm{cm}^{-2}$. After ion implantation, the substrate undergoes another annealing process (at $1,100^{\circ} \mathrm{C}$ in air for $8 \mathrm{~h}$ ) for eliminating the surface defects. Subsequently, the growth process of SWNT arrays followed the same procedure as mentioned above.

Characterization of high-density SWNT arrays. The as-grown SWNTs were inspected with SEM (Hitachi S4800 field emission, Japan), AFM (Veeco NanoScope IIIA, Veeco Co.) and a Raman spectrometer (Horiba HR800 Raman system) to characterize the morphology, structure and electrical transport properties. XPS (ESCALab250, Thermo Scientific Corporation) and AFM were used to confirm the chemical composition and morphology of the substrate surface.

Fabrication and measurements of electrical devices. Devices were fabricated through a self-aligned U-gate process ${ }^{30,31}$. A bilayer e-beam resist with continuously varying sensitivity was used for defining the U-shaped top-gate stack, after electron-beam lithography and develop, $12 \mathrm{~nm} \mathrm{HfO}_{2}$ and $70 \mathrm{~nm}$ Ti were sequentially deposited by atomic layer deposition and electron-beam evaporation 
followed with a traditional lift-off process. The pad of the source, drain and gate was composed of $\mathrm{Ti} / \mathrm{Pd} / \mathrm{Au} 0.5 / 30 / 50 \mathrm{~nm}$, and the channel was defined through reactive ion etching using oxygen plasma. Ten nanometres of Pd was finally deposited as a self-aligned contact layer.

The d.c. characteristics of our devices were measured in a vacuum cryogenic probe station (Lakeshore TTP4) with a semiconductor analyzer (Keithley 4200 SCS). The a.c. responses of our FETs were characterized by S-parameter measurements and it was carried out on a microwave probe station with GSG probes (Cascade 9000) in atmosphere. The de-embedding process was based on a short-open-load-through scheme.

\section{References}

1. Avouris, P., Chen, Z. \& Perebeinos, V. Carbon-based electronics. Nat. Nanotech. 2, 605-615 (2007).

2. Che, Y. et al. Review of carbon nanotube nanoelectronics and macroelectronics. Semicond. Sci. Technol. 29, 073001 (2014).

3. Franklin, A. D. Electronics: the road to carbon nanotube transistors. Nature 498, 443-444 (2013).

4. Ding, L., Yuan, D. \& Liu, J. Growth of high-density parallel arrays of long single-walled carbon nanotubes on quartz substrates. J. Am. Chem. Soc. 130, 5428-5429 (2008).

5. Wang, C. et al. Synthesis and device applications of high-density aligned carbon nanotubes using low-pressure chemical vapor deposition and stacked multiple transfer. Nano Res. 3, 831-842 (2010).

6. Li, X. et al. Langmuir - Blodgett assembly of densely aligned single-walled carbon nanotubes from bulk materials. J. Am. Chem. Soc. 129, 4890-4891 (2007).

7. Cao, Q. et al. Arrays of single-walled carbon nanotubes with full surface coverage for high-performance electronics. Nat. Nanotech. 8, 180-186 (2013).

8. Hong, S. W., Banks, T. \& Rogers, J. A. Improved density in aligned arrays of single-walled carbon nanotubes by sequential chemical vapor deposition on quartz. Adv. Mater. 22, 1826-1830 (2010)

9. Zhou, W., Ding, L., Yang, S. \& Liu, J. Synthesis of high-density, large-diameter, and aligned single-walled carbon nanotubes by multiple-cycle growth methods. ACS Nano 5, 3849-3857 (2011).

10. $\mathrm{Wu}, \mathrm{B}$. et al. Ultrahigh density modulation of aligned single-walled carbon nanotube arrays. Nano Res. 4, 931-937 (2011)

11. Chen, Y. \& Zhang, J. Chemical vapor deposition growth of single-walled carbon nanotubes with controlled structures for nanodevice applications. Acc. Chem. Res. 47, 2273-2281 (2014).

12. Chen, Y. et al. State of the art of single-walled carbon nanotube synthesis on surfaces. Adv. Mater. 26, 5898-5922 (2014).

13. Li, Y. et al. How catalysts affect the growth of single-walled carbon nanotubes on substrates. Adv. Mater. 22, 1508-1515 (2010).

14. Zhou, W., Ding, L. \& Liu, J. Role of catalysts in the surface synthesis of single-walled carbon nanotubes. Nano Res. 2, 593-598 (2009).

15. Ago, H., Nakamura, Y., Ogawa, Y. \& Tsuji, M. Combinatorial catalyst approach for high-density growth of horizontally aligned single-walled carbon nanotubes on sapphire. Carbon 49, 176-186 (2011).

16. Ago, H. et al. Synthesis of horizontally-aligned single-walled carbon nanotubes with controllable density on sapphire surface and polarized Raman spectroscopy. Chem. Phys. Lett. 421, 399-403 (2006).

17. He, M. et al. Iron catalysts reactivation for efficient CVD growth of swnt with base-growth mode on surface. J. Phys. Chem. B 108, 12665-12668 (2004).

18. Ago, H., Ayagaki, T., Ogawa, Y. \& Tsuji, M. Ultrahigh-vacuum-assisted control of metal nanoparticles for horizontally aligned single-walled carbon nanotubes with extraordinary uniform diameters. J. Phys. Chem. C 115, 13247-13253 (2011).

19. Ning, G. et al. Improvement of $\mathrm{Fe} / \mathrm{MgO}$ catalysts by calcination for the growth of single- and double-walled carbon nanotubes. J. Phys. Chem. B 110, 1201-1205 (2005).
20. Xie, H. et al. Growth of high-density parallel arrays of ultralong carbon nanotubes with catalysts pinned by silica nanospheres. Carbon 52, 535-540 (2013).

21. Zhang, R. et al. The reason for the low density of horizontally aligned ultralong carbon nanotube arrays. Carbon 52, 232-238 (2013).

22. Li, J. et al. Direct identification of metallic and semiconducting single-walled carbon nanotubes in scanning electron microscopy. Nano Lett. 12, 4095-4101 (2012).

23. Hong, G. et al. Direct growth of semiconducting single-walled carbon nanotube array. J. Am. Chem. Soc. 131, 14642-14643 (2009).

24. Hong, G. et al. Separation of metallic and semiconducting single-walled carbon nanotube arrays by 'scotch tape'. Angew. Chem. Int. Ed. 50, 6819-6823 (2011).

25. Petit, P. et al. Study of the thermal stability of supported catalytic nanoparticles for the growth of single-walled carbon nanotubes with narrow diameter distribution by chemical vapor deposition of methane. J. Phys. Chem. C 116, 24123-24129 (2012).

26. McHargue, C. J., Ren, S. X., Sklad, P. S., Allard, L. F. \& Hunn, J. Preparation of nanometer-size dispersions of iron in sapphire by ion implantation and annealing. Nucl. Instrum. Methods Phys. Res. B 116, 173-177 (1996).

27. Rutherglen, C., Jain, D. \& Burke, P. Nanotube electronics for radiofrequency applications. Nat. Nanotech. 4, 811-819 (2009).

28. Steiner, M. et al. High-frequency performance of scaled carbon nanotube array field-effect transistors. Appl. Phys. Lett. 101, 053123 (2012).

29. Franklin, A. D., Lin, A., Wong, H. S. P. \& Zhihong, C. Current scaling in aligned carbon nanotube array transistors with local bottom gating. Electron Dev. Lett. IEEE 31, 644-646 (2010).

30. Ding, L. et al. Self-aligned U-gate carbon nanotube field-effect transistor with extremely small parasitic capacitance and drain-induced barrier lowering. ACS Nano 5, 2512-2519 (2011).

31. Zhang, Z. et al. Self-aligned ballistic $\mathrm{n}$-type single-walled carbon nanotube fieldeffect transistors with adjustable threshold voltage. Nano Lett. 8, 3696-3701 (2008).

\section{Acknowledgements}

We are grateful to Dr Liming Xie (National Center for Nanoscience and Nanotechnology, China) and Rong Jian (Peking University, China) for their helpful discussions about scanning electronic microscopy. Y.H. and J.Z. also thank Yanlian Yang (National Center for Nanoscience and Nanotechnology, China) for her help with atomic force microscopy. This work was supported by the Beijing Municipal Science and Technology Commission (D141100000614001), NSFC (21233001, 21129001, 51272006, 51432002 and 51121091) and MOST (2011CB932601).

\section{Author contributions}

J.Z., Y.H., L.K., Q.Z. and Z.L. designed the experiment. Y.H., L.K., Q.Z., S.Z., L.Y. and Z.W. performed the experiments and analysed the results. H.Z. and Z.Z. performed the electrical measurement. J.Z., Y.H., L.K., Q.Z., H.Z., J.L., Q.L., Z.Z., L.P. and Z.L. contributed to the writing and discussion of the manuscript.

\section{Additional information}

Supplementary Information accompanies this paper at http://www.nature.com/ naturecommunications

Competing financial interests: The authors declare no competing financial interests.

Reprints and permission information is available online at http://npg.nature.com/ reprintsandpermissions/

How to cite this article: Hu, Y. et al. Growth of high-density horizontally aligned SWNT arrays using Trojan catalysts. Nat. Commun. 6:6099 doi: 10.1038/ncomms7099 (2015). 